\title{
Defining Theoretical Limits of Aberration-Corrected Electron Tomography: New Bounds for Resolution, Object Size, and Dose
}

\author{
Reed Yalisove ${ }^{1}$, Suk Hyun Sung ${ }^{1}$ and Robert Hovden ${ }^{1,2}$ \\ 1. Department of Materials Science and Engineering, University of Michigan, Ann Arbor, MI, USA. \\ 2. Applied Physics Program, University of Michigan, Ann Arbor, MI, USA.
}

The theoretical limits of electron tomography have long been defined by the Crowther criterion, which relates $3 \mathrm{D}$ resolution to the number of projections acquired [1], and the dose fractionation theorem [2], which dictates total dose requirement across projections. However, these relations are invalid for aberration-corrected scanning transmission electron microscopy (STEM) where high convergence angles limit the ability to reconstruct objects larger than the depth-of-focus (c.a. $5 \mathrm{~nm}$ ). Hovden, et al. showed overcoming the limitations of aberration-corrected STEM tomography requires collecting information beyond a traditional tilt series by acquiring a through-focal stack at every tilt [3]. Here, information is no longer mapped to a plane in Fourier (k) space, but becomes a volumetric toroid [4]. Although experimentally demonstrated, the theoretical limits of aberration-corrected tomography remain undefined. Understanding the limits of resolution, object size, and dose using highly-convergent beams requires new theoretical approaches.

Here we present a theoretical foundation for aberration-corrected electron tomography by establishing analytic descriptions for resolution, sampling, object size, and dose-with direct analogy to the Crowther criterion. Figure 1a,b shows the 3D structure of a contrast transfer function (CTF) for through focal tomography where every specimen tilt measures a toroid with petal-shaped cross-section (Fig. 1c). A remarkable feature of the 3D CTF is the overlapped regions that permit complete information collection (Fig. 2a,b) - unachievable with conventional tomography. This breaks expected Crowther relationships and the maximum reconstructable object size is unlimited up to spatial frequency $k_{c}$. At resolutions beyond $2 / k_{c}$, Crowther-like tradeoffs define the maximum object size (D) allowed for given $3 \mathrm{D}$ resolution $(d)$ :

$$
k_{c}=\frac{2 \alpha-\Delta \theta}{\lambda} \cdots(1) \quad \mathrm{D}=\left\{\begin{array}{ll}
\frac{d^{2}}{\lambda\left(2-k_{c} d\right)}, & \frac{\lambda}{\alpha}<d<\frac{2}{k_{c}} \\
\infty, & \frac{2}{k_{c}} \leq d<\infty
\end{array} \quad \cdots\right. \text { (2) }
$$

where $k_{c}$ is the maximum frequency allowed for complete information transfer, $\alpha$ is convergence semi-angle, $\Delta \theta$ is tilt increment, and $\lambda$ is electron wavelength. As shown in Figure 2, when the tilt angle spacing becomes smaller than $2 \alpha$ a continuum of information is measured and object size is unbounded at midband resolution. This occurs under typical instrument operation $(>25 \mathrm{mrad})$ and sampling $\left(<2^{\circ}\right.$ tilt $)$. For smaller specimen tilt increments, resolution improves and allowed object size increases (Fig. 2c).

Despite the large amount of image data required by aberration-corrected tomography of nanoscale materials, dose can be distributed to mitigate the total specimen exposure. Extending the dose fractionation arguments presented by Hoppe [2] and Saxton [5], we show aberration-corrected electron tomography allows tunable dose allocation between images at every tilt and the total number of tilts.

These results have critical significance for the next generation of electron microscopes with ever increasing convergence angles (> $60 \mathrm{mrad})$.

References:

[1] R Crowther, D DeRosier and A Klug, Proc. R. Soc. A 317 (1970), p. 319.

[2] R Hegerl and W Hoppe, Zeitschrift für Naturforschung A 31(12) (1976), p. 1717.

[3] R Hovden et al., Ultramicroscopy 140 (2014), p. 26.

[4] V Intaraprasonk, H Xin and D Muller, Ultramicroscopy 108 (2008), p. 1454. 
[5] B Saxberg and W Saxton, Ultramicroscopy 6 (1981), p. 85.

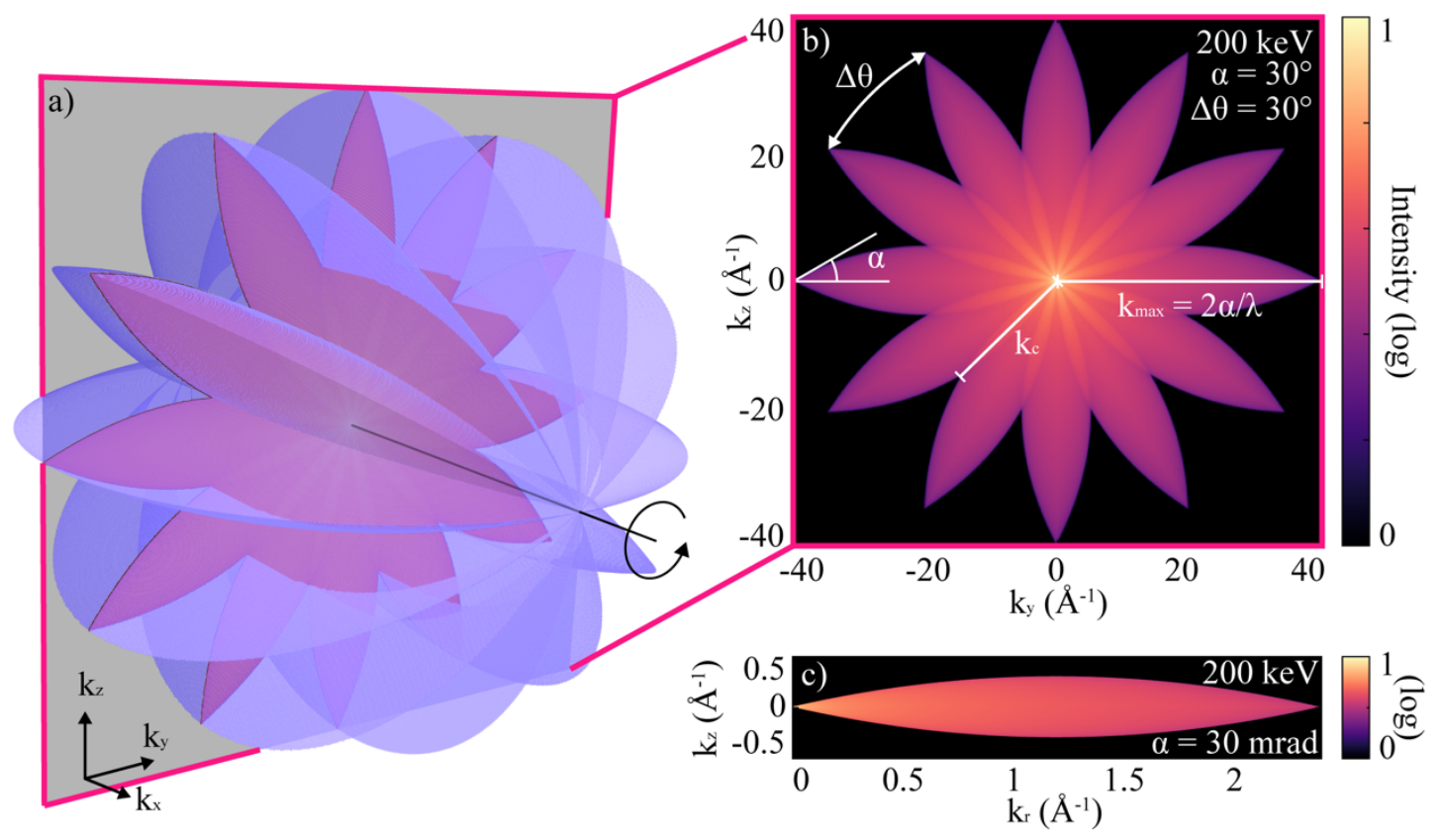

Figure 1. a) $3 \mathrm{D}$ CTF of through-focal tomography (tilt axis, $\hat{x}$ ). Blue shell denotes the information transfer limit in 3D. $\alpha$ and $\Delta \theta$ are exaggerated to $30^{\circ}$. The plane slices through the CTF at $\mathrm{k}_{\mathrm{x}}=0 . \mathrm{b}$ ) Internal structure of the CTF at $\mathrm{k}_{\mathrm{x}}=0$ is highlighted. Each petal-shaped lobe represents a single through-focal CTF. Complete transfer of information is guaranteed within a spherical radius $k_{c}$. c) Through-focal CTF for typical aberrationcorrected STEM $(\alpha=30 \mathrm{mrad}, 200 \mathrm{keV})$.
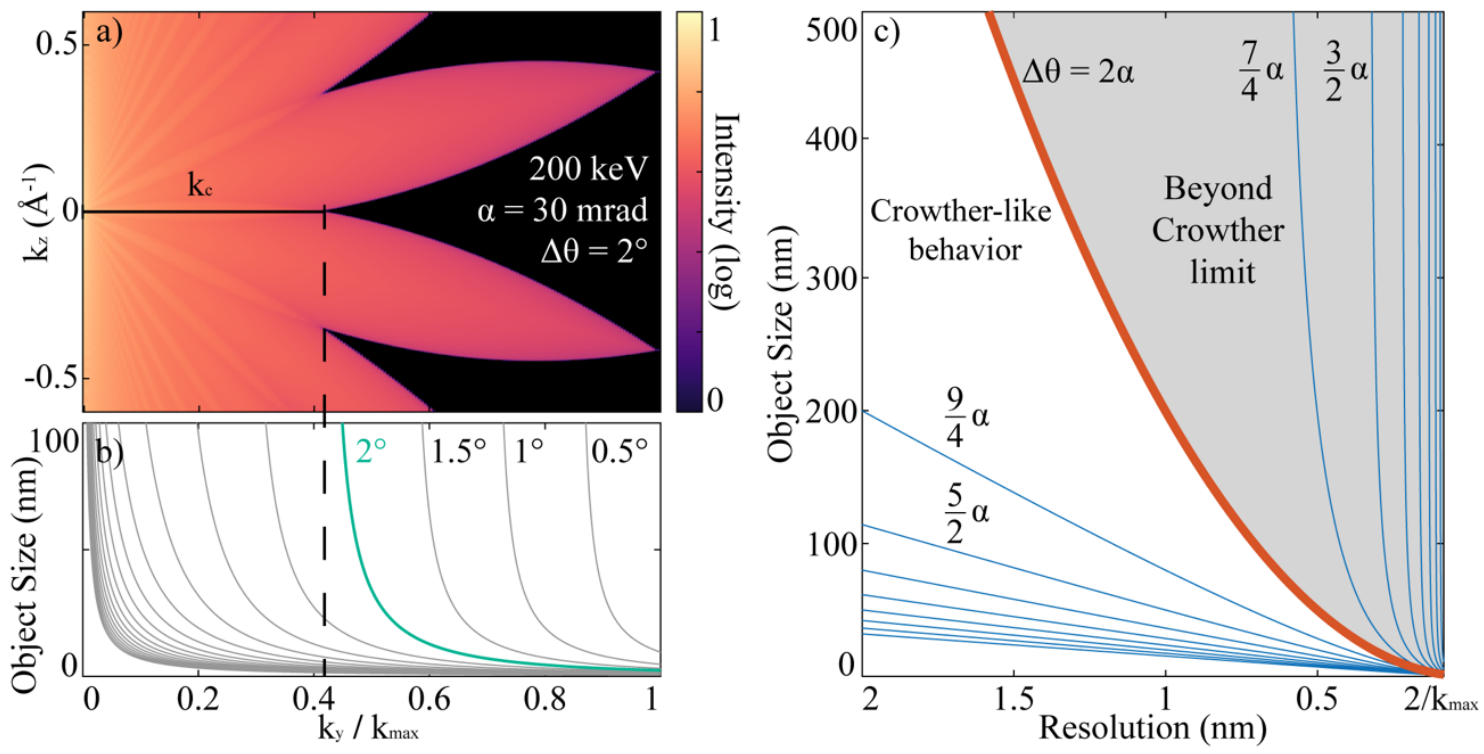

Figure 2. The CTF of through-focal series hosts overlap regions which permit complete information transferand therefore unlimited object size. a) CTF in cross-section for $200 \mathrm{keV}, 30 \mathrm{mrad}, 2^{\circ}$ tomography. $\mathrm{k}_{\mathrm{c}}$ denotes maximum frequency of complete information transfer. b) Spatial frequency vs. maximum object size for several tilt step-sizes with teal curve matching conditions in a). For $\mathrm{k}<\mathrm{k}_{\mathrm{c}}$, the maximum object size is unbounded. c) Resolution vs. maximum object size. Tradeoff between resolution and object size surpasses the Crowther limit, particularly when $\Delta \theta \leq 2 \alpha$ (grey) and objects of any size may be reconstructed. 